\title{
The impact of the COVID-19 pandemic on a cohort of Labrador Retrievers in England
}

\author{
Charlotte S C Woolley ( $\nabla$ s1576473@sms.ed.ac.uk) \\ The Roslin Institute and Royal (Dick) School of Veterinary Studies, University of Edinburgh \\ lan G Handel \\ The Roslin Institute and Royal (Dick) School of Veterinary Studies, University of Edinburgh \\ B Mark Bronsvoort \\ The Roslin Institute and Royal (Dick) School of Veterinary Studies, University of Edinburgh \\ Jeffrey J Schoenebeck \\ The Roslin Institute and Royal (Dick) School of Veterinary Studies, University of Edinburgh \\ Dylan N Clements \\ The Roslin Institute and Royal (Dick) School of Veterinary Studies, University of Edinburgh
}

\section{Research Article}

Keywords: Epidemiology, Dog health, Dog lifestyle, Veterinary care, Pandemic, COVID-19 restrictions, Cohort, Longitudinal data, Labrador Retriever, Questionnaires

Posted Date: February 11th, 2022

DOI: https://doi.org/10.21203/rs.3.rs-1238396/v1

License: (c) (1) This work is licensed under a Creative Commons Attribution 4.0 International License. Read Full License 


\section{Abstract}

\section{Background}

The COVID-19 pandemic is likely to have affected the welfare and health of dogs, due to surges in adoptions and purchases, changes in the physical and mental health and financial status of dog owners, changes in dogs' lifestyle and routines and limited access to veterinary care. Research is needed to be able to assess the extent of these effects. The aims of this study were to investigate whether COVID-19 restrictions were associated with differences in Labrador Retrievers' lifestyle, routine care, insurance status, illness incidence or veterinary attendance with an illness, who were living in England and enrolled in Dogslife, an owner-based cohort study. Longitudinal questionnaire data from Dogslife that was relevant to the dates between the $23^{\text {rd }}$ of March and the $4^{\text {th }}$ of July 2020, during COVID-19 restrictions in England, were compared to data between the same dates in previous years from 2011 to 2019 using mixed regression models and adjusted chi-squared tests.

\section{Results}

The COVID-19 restrictions study period (March 23rd to July 4th 2020) was associated with owners who enrolled in Dogslife reporting increases in dogs' exercise and worming and decreases in insurance, titbit-feeding and vaccination, in comparison with previous years (March 23rd to July 4th, 2010 to 2019). There were decreased odds of owners reporting that their dogs had an episode of coughing $(0.20,95 \% \mathrm{Cl}: 0.04-0.92)$ and that they took their dog to a veterinarian with an episode of any illness $(0.58,95 \% \mathrm{Cl}: 0.45-0.76)$ during the COVID-19 restrictions and owners were even less likely to take their dog to a veterinarian with certain illnesses.

\section{Conclusions}

Dogslife provided a unique opportunity to study prospective questionnaire data from owners already enrolled on a longitudinal cohort study, which minimised bias associated with recalling events prior to the pandemic, allowed a wider population of dogs to be studied than is available from primary care data and offered unique insights into owners' decision making about their dogs' healthcare. There are clear implications of the COVID-19 pandemic and associated restrictions for the lifestyle, care and health of dogs.

\section{Background}

The emergence of a novel coronavirus (SARS-CoV-2) in December 2019 and the subsequent COVID-19 pandemic that swept across nations worldwide led governments in many countries to impose social restrictions to control the spread of the virus $(1,2)$. In the UK, a national lockdown was implemented on the $23^{\text {rd }}$ of March 2020, where people were not permitted to leave their homes without a reasonable excuse (3). These strict rules were eased over subsequent months, but the long-term impact of the pandemic itself and these associated control measures are not yet fully understood. In 2021, the Pet Food Manufacturers' Association (PFMA) estimated that there were 12.5 million dogs in the UK, with one third of households owning a dog (4). Aspects of the COVID-19 pandemic that are likely to have affected the welfare of pet dogs include surges in pet adoptions and purchases (5-7), the physical and mental health of their owners, changes in their lifestyle and routine, the financial status of their owners and limited access to veterinary care $(8,9)$.

People with COVID-19 may be bedridden for weeks $(10,11)$ and unable to give their pets the quality of care that they usually receive. When dog owners die, are hospitalised or are forced to isolate, dogs may need to adapt to stressful new environments, people and routines $(12,13)$. Many owners did not have a provisional care plan for their pet during the initial months of the pandemic, which may have led to sub-optimal care $(14,15)$. Several mental health implications of the COVID-19 lockdown restrictions have been reported (16-18). Pet ownership during the pandemic has mostly been associated with positive mental health outcomes (19) but some owners felt concerned or stressed about caring for their pet (20-22) or felt their relationship with their pet became strained $(19,22)$. The stress levels, emotional and physiological states of dog owners affect the stress levels, cognitive performance, and quality of life of their dogs (23-27) and owners with poorer mental health after lockdown reported more changes in their pets' behaviour and welfare, both negative and positive (28).

COVID-19 lockdown restrictions in the UK led to a reduction in social interactions and differences in physical activity (29-33) and many people were furloughed or worked from home (34). Several surveys have reported changes to dogs' routines, including decreased time left alone, differences in walking patterns and training and fewer opportunities to socialise with other dogs (15,35-40). Due to the strong attachment bond of pet dogs and their owners (41), increased contact would initially seem like a positive change, especially if they are usually left alone for extended time periods (42). However, increased exposure to physical contact and decreased time exercising outside and socialising may exasperate behavioural difficulties in adult dogs or increase the chance of behavioural problems in puppies (43). Several surveys have reported negative changes in dogs' behaviour during COVID-19 lockdown restrictions $(15,39,44-47)$ and an increase in the frequency and severity of dog bites was seen in paediatric departments during the 2020 lockdown in Italy (48).

Veterinary practices were exempt from closing during the first UK lockdown but non-essential care was postponed $(43,49)$. The pandemic might also have caused financial difficulty for some pet owners, meaning they could not afford veterinary bills or pet insurance $(43,50)$. Results from a RCVS survey showed that in April $2020,95 \%$ of veterinary practices saw a reduction in weekly practice turnover of $25 \%$ or more, $66 \%$ saw a reduction of $50 \%$ or more and $24 \%$ saw a reduction of $75 \%$ or more (51). The Small Animal Veterinary Surveillance Network (SAVSNET) reported reductions in vaccination consultations during the first 2020 lockdown period and subsequent peaks in vaccine-controlled diseases such as parvovirus and leptospirosis. Reductions in consultations for gastroenteric and respiratory clinical signs, pruritis, trauma and tumours were also reported (52-54).

Research has highlighted the problem of the "symptom iceberg" in medical consultation data: the missing information about the wider population that does not exist in healthcare records (55). During a recent vomiting outbreak in dogs in the UK, the vomiting incidence rates reported by Dogslife, an owner-based 
longitudinal cohort study, were over double the equivalent vomiting consultation rates reported by SAVSNET $(56,57)$. This can be explained by Dogslife reports showing that only one third of vomiting episodes led to a veterinary consultation (58). Therefore, with medical consultation data it is impossible to distinguish whether reduced numbers of veterinary consultations were indicative of decreased illness incidence due to some protective measures of lockdown restrictions, fewer owners taking their dogs to the veterinarian with illness symptoms, or a combination. Furthermore, most research into changes in dogs' lifestyles and routines during COVID-19 restrictions has been based on cross-sectional prospective surveys, which do not necessarily accurately estimate their extent. To the author's knowledge, no studies have reported on routine care, such as worming and treatment with anti-parasitic therapeutics and further research is needed to understand the implications of lifestyle changes on dogs' physical health. Longitudinal cohorts established prior to the pandemic, such as Dogslife (59), provide an opportunity to study both lifestyle factors and illness incidence within the wider population before and after the pandemic, and thus, provide a more accurate estimation of the extent and impact of changes that occurred.

The first aim of this study was to investigate whether COVID-19 restrictions were associated with differences in Dogslife Labrador Retrievers' lifestyle (including exercise, dietary factors, bathing and sleeping habits), routine care (including worming, anti-parasitic treatments for fleas and ticks and vaccination) or insurance status. The second aim was to investigate whether COVID-19 restrictions were associated with differences in Dogslife Labrador Retrievers' illness incidence or veterinary attendance with an illness.

\section{Results}

\section{The association of COVID-19 restrictions with the lifestyle and routine care of Dogslife dogs}

To investigate the association of the COVID-19 restrictions with the lifestyle and routine care of Dogslife dogs, variables of interest from 13716 questionnaires from 3889 dogs, entered into the Dogslife website by owners between March $23^{\text {rd }}$ and July $4^{\text {th }}, 2011$ to 2020 were included in analysis. Summary statistics of the dogs in the questionnaires are given in Table 1. The data available from the questionnaires and the estimated beta coefficients and odds ratios for comparing the COVID-19 restrictions study period (March 23rd to July $4^{\text {th }}$ 2020) to data in the same date range in previous years (March 23rd to July $4^{\text {th }}, 2010$ to 2019) from the linear and logistic generalised additive mixed effects models (GAMMs) respectively, adjusted for age, sex and individual effects for each the variables of interest are reported in the forest plot in Figure 1.

Table 1: Summary statistics of Dogslife dogs $(N=3889)$

\begin{tabular}{|c|c|c|}
\hline Variable & $\begin{array}{l}\text { COVID-19 restrictions study period (March 23rd to July } \\
4^{\text {th }} 2020 \text { ) }\end{array}$ & $\begin{array}{l}\text { Same date range in previous years (March 23rd to July } 4^{\text {th }}, 2010 \\
\text { to 2019) }\end{array}$ \\
\hline $\begin{array}{l}\text { Number of } \\
\text { questionnaires }\end{array}$ & 1265 & 12451 \\
\hline \multicolumn{3}{|l|}{ Age category } \\
\hline Under 1 year & $124(9.80 \%)$ & $5308(42.63 \%)$ \\
\hline 1 to 3.49 years & $219(17.31 \%)$ & 3994 (32.08\%) \\
\hline 3.5 to 6.99 years & 418 (33.04\%) & 2521 (20.25\%) \\
\hline 7 or over & 504 (39.84\%) & $628(5.04 \%)$ \\
\hline \multicolumn{3}{|l|}{ Sex } \\
\hline Female & $646(51.07 \%)$ & $6134(49.27 \%)$ \\
\hline Male & $619(48.93 \%)$ & $6317(50.73 \%)$ \\
\hline \multicolumn{3}{|l|}{ Working status } \\
\hline Pet only & 1155 (91.38\%) & $11043(90.52 \%)$ \\
\hline $\begin{array}{l}\text { Working/sporting/guide } \\
\text { dog }\end{array}$ & $109(8.62 \%)$ & $1157(9.48 \%)$ \\
\hline
\end{tabular}

Dog age was calculated to the date that owners entered questionnaire data into the Dogslife website

The COVID-19 restrictions study period was associated with owners reporting an increase of 6.89 minutes per week ( $95 \%$ Cl: $1.65-12.13)$ in the quantity of exercise dogs received and increased odds that they reported their dogs were treated with a wormer since the owner's last visit to the Dogslife website (SLV) $(1.20,95 \% \mathrm{Cl}: 1.02-1.40)$ and decreased odds that they reported their dogs were insured (0.59, 95\% Cl: 0.39 - 0.90), were given titbits (0.36, $95 \% \mathrm{Cl}: 0.24-$ $0.52)$ and were vaccinated SLV $(0.70,95 \% \mathrm{Cl}: 0.58-0.83)$. The COVID-19 restrictions study period was not associated with owners reporting a difference in the quantity of dried food that dogs were fed or a change in odds of owners reporting their dogs sleep with a person at night, were bathed SLV or treated with an anti-parasitic SLV. An additional file provides further details of the models' selection, diagnostics, fit and full output [see Additional file 1].

\section{The association of COVID-19 restrictions with illness incidence and associated veterinary attendance in Dogslife}

To investigate the association of the COVID-19 restrictions with Dogslife illness incidences and veterinary attendance with illness incidences, illness data from 16115 questionnaires from 4110 dogs, entered into the Dogslife website by owners between March $23^{\text {rd }}$ and July $23^{\text {rd }}, 2011$ to 2020 were included in 
analysis. Of these questionnaires, 3320 recorded at least one incidence of illness and 1850 recorded at least one incidence where the owner had taken their dog to the veterinarian with an illness. The Mantel-Haenszel (MH) adjustment was performed in chi-squared tests for illness incidences and veterinary attendance with illness incidences with any illness and each illness type individually, except for veterinary attendance with coughing and accident or injury, due to expected frequencies of the stratified groups containing values $<5$. The estimated odds ratios for comparing Dogslife illness incidences and veterinary attendance with illness incidences in the COVID-19 restrictions study period to data in the same date range in previous years from chi-squared tests for any illness and each illness type individually were estimated and are reported in the forest plot in Figure 2.

The COVID-19 restrictions study period was associated with decreased odds of owners reporting that their dogs had an episode of coughing (0.20, $95 \%$ Cl: $0.04-0.92$ ) but was not associated with a change in odds of owners reporting that their dogs had an episode of any illness, vomiting, diarrhoea, scratching, licking or chewing, limping or lameness, ear problems, eye problems, skin problems or accident or injury. The COVID-19 restrictions study period was associated with decreased odds of owners reporting that they took their dog to a veterinarian with an episode of any illness (0.58, $95 \% \mathrm{Cl}$ : $0.45-0.76)$, scratching $(0.46,95 \% \mathrm{Cl}: 0.23-0.96)$, limping or lameness $(0.56,95 \% \mathrm{Cl}: 0.33-0.97)$, eye problems $(0.12,95 \% \mathrm{Cl}$ : $0.02-0.94)$ and accident of injury $(0.17$, $95 \% \mathrm{Cl}: 0.03-0.90)$ but was not associated with a change in odds of owners reporting that their dogs had an episode of vomiting, diarrhoea, coughing, licking or chewing, ear problems or skin problems.

\section{Discussion}

This study established that the COVID-19 restrictions study period was associated with differences in Labrador Retrievers' lifestyle, routine care, insurance status, illness incidence and veterinary attendance for those dogs who were living in England and enrolled in Dogslife. To our knowledge, this is the first time data from a cohort study has been used to investigate associations between COVID-19 restrictions and canine lifestyles, health and veterinary care.

This study estimates that Dogslife owners reported that they exercised their dogs for an average 6.89 (95\% Cl: 1.65 - 12.13) minutes longer per week after the COVID-19 restrictions study period. This result initially seems in contrast with surveys of dog owners internationally, where dog owners reported their dogs had fewer walks (39), in Belgrade, where the number of minutes dog owners spent walking their dogs decreased (36) and in the UK, where dogs were walked less frequently and closer to home, but the number of minutes they were exercised for remained the same (15). However, the exercise reported in Dogslife was transformed from a categorical to a continuous variable prior to analysis and averaged across several different types of exercise. Research from Dogs Trust reported that dog owners played with or trained their dogs more frequently in lockdown, but the number of minutes and frequency of walks decreased and more time was spent walking on a lead, although the effect of breed was not accounted for (40). Therefore, it could be that Dogslife Labrador Retrievers were indeed walked less or similar amounts, but owners compensated with other forms of exercise due to spending more time with their dogs at home.

While to date there has been no peer reviewed research into dogs' diet during lockdown, there have been several reports in the media of dog owners titbitfeeding more frequently during lockdown. The pet food brand Natural Instinct reported that $44 \%$ of the dog owners they surveyed gave their dogs more treats during lockdown (60), over half of owners surveyed by the charity Guide Dogs reported giving their dogs more treats (61) and $34 \%$ of dog owners surveyed by insurance group More Than reported giving their pets more treats (62). These surveys are in contrast with results from this study, which found decreased odds $(0.36,95 \% \mathrm{Cl}: 0.24-0.52)$ of owners reporting that they fed their dogs titbits SLV during the COVID-19 restrictions study period. An explanation is that a large proportion of Dogslife dogs were working, sporting or guide dogs: $9.48 \%$ prior to and $8.62 \%$ during the COVID-19 restrictions study period (Table 1 ). Owners would not have been able to take their dogs to sporting activities or training classes during lockdown, which may have reduced the number of titbits given as rewards. In support of this theory, one survey of 1833 Italian dog owners reported that $26 \%$ gave their dogs treats as a reward during training and sports activities (63) and a qualitative analysis of another survey in the UK revealed that some owners felt that treats should only be given as part of training (64). However, as Dogslife does not collect information on the reasons behind titbit-feeding or how regularly both pets and working, sporting or guide dogs are taken to training classes, it is impossible to infer whether a reduction in these activities was likely to cause such a large drop in the odds of titbit-feeding by owners.

The odds $(1.20,95 \%$ Cl: $1.02-1.40)$ of owners reporting they had wormed their dog SLV increased during the COVID-19 restrictions study period. Despite the fact that many veterinary practices had limited services, they were still able to dispense wormers for dog owners to collect (49). Furthermore, one survey reported that only $69.3 \%$ of dog owners in the UK sought advice from their veterinarian about worming (65), so a large proportion of dog owners may have not been affected by changes in veterinary services in terms of their dogs' worming routines. The higher rates of worming could be explained by improved compliance of owners due to having more time at home with their dogs and being less likely to forget (66). The results of this study are in contrast with reports of increased cases of Angiostrongylus vasorum lungworm after COVID-19 restrictions began in the UK, which was considered to be a result of a reduction in routine worming treatments (67).

A UK-based survey reported that people who bought puppies during the pandemic were more likely to be first-time pet owners and were more likely to buy from breeders who did not perform health checks on the puppies before sale (7). Therefore, a rise in inexperienced dog owners who bought from disreputable breeders may have exasperated the problem of poor parasite control measures. New puppy owners may have been reluctant to take them to a veterinarian in the pandemic and may not have received a parasite prevention plan (67). The demographics of Dogslife dog owners are considerably different: they buy Kennel Club registered Labrador Retrievers and a proportion of Dogslife dogs have another purpose in addition to being pets. Furthermore, $90.2 \%$ of dogs in the COVID-19 restrictions study period were over 1 year of age (Table 1), so most owners had at least some experience of dog ownership and would have probably already been in the routine of parasite prevention.

It is not surprising that there were reduced odds $(0.70,95 \% \mathrm{Cl}: 0.58-0.83)$ of Dogslife owners reporting that they had vaccinated their dog SLV during the COVID-19 restrictions study period. There was some confusion about the guidelines from the British Veterinary Association (BVA) about whether veterinary

Page $4 / 12$ 
practices should administer vaccinations during the first 2020 lockdown period and while most ceased to administer them, some continued to prevent their emergency services being overwhelmed (68). The results reported here mirror the reduction in canine vaccination consultations reported by SAVSNET, who reported that the percentage change in vaccination consultation frequency in dogs during the first social distancing phase of lockdown (23rd March - 12th April 2020) compared to the same time period in 2019 approached approximately $-95 \%$ and remained at around $-40 \%$ by the $4^{\text {th }}$ of July 2020 ( 69 ). Perhaps what is more pertinent is the implications of these results. The WHO have recently warned of the risk of measles outbreaks worldwide due to children missing their routine vaccines during the COVID-19 pandemic (70). In canine medicine, rises in cases of vaccine preventable diseases such of parvovirus and leptospirosis after the 2020 lockdown reported by SAVSNET are small yet concerning. Although these diseases currently seem under control, it is not clear whether there will be long-term consequences in immunity for puppies who missed their vaccinations within the optimal time frames, which are recommended to ensure that they are capable of making a primary immune response (71).

The odds $(0.36,95 \% \mathrm{Cl}: 0.24-0.52)$ of owners reporting that their dogs were insured were decreased during the COVID-19 restrictions study period, which could be linked to the financial status of owners. The BVA suggested that some owners may deal with the financial pressure of the pandemic in the shortterm by cancelling their pet insurance (43). Furthermore, the price comparison site GoCompare reported a $26 \%$ increase in the price of dog insurance in May 2020 in comparison with May 2019, which may have made the decision to cancel or avoid renewing dogs' insurance easier for some pet owners (72).

The illness incidence of Dogslife dogs did not change during the COVID-19 restrictions study period. However, as this study only included data between the $23^{\text {rd }}$ of March and the $4^{\text {th }}$ of July that were recorded up to a maximum of the $23^{\text {rd }}$ of July in each year, it is possible that the long-term health of dogs was not yet affected. On the other hand, the odds of owners reporting that their dog experience a coughing episode during the COVID-19 restrictions study period was greatly reduced to a fifth of previous years $(0.20,95 \% \mathrm{Cl}: 0.04-0.92)$. A common cause of coughing in dogs is canine infectious respiratory disease, also known as "Kennel cough", which transmits between dogs. has multiple viral and bacterial pathogens responsible for its causation and is endemic in the population $(73,74)$. It is probable that the COVID-19 restrictions led to a reduction in dog socialisation and therefore the transmission of pathogens and that a subsequent reduction in coughing was reported by owners.

The odds of owners reporting that they took their dog to a veterinarian with an illness episode during the COVID-19 restrictions study period were reduced in comparison with previous years $(0.58,95 \% \mathrm{Cl}: 0.45-0.76)$. When specific illnesses were examined, the reduction in odds was still apparent in owners reporting that they took their dog to a veterinarian with scratching $(0.46,95 \% \mathrm{Cl}: 0.23-0.96)$, limping and lameness $(0.56,95 \% \mathrm{Cl}$ : $0.33-0.97)$, eye problems (0.12, 95\% Cl: $0.02-0.94)$ and accident or injury (not adjusted for age) $(0.17,95 \% \mathrm{Cl}: 0.03-0.90)$. The RCVS reported that in April $2020,97 \%$ of practices it surveyed had limited their services to emergencies and 'urgent cases', although how these were defined by different practices were likely to differ and a further $29 \%$ had closed either a branch or their main practice (51). Owners were probably less likely to take their dog to the veterinarian when they perceived their dog had a milder problem, such as many cases of scratching or eye problems. They may have also been able to pick up their dogs' repeat prescriptions for chronic or repetitive illnesses, such as many cases of limping or lameness. Accidents or injuries indicate more immediate problems which may require urgent veterinary treatment. However, this study did not differentiate between mild or more serious types of illness or identify chronic illnesses, so it is impossible to comment whether this was the case.

Alternatively, owners may have had a telephone consultation with their veterinarian about these complaints. In Dogslife, owners are asked "Did you take [dog name] to your vet for the [illness type]?" rather than if the owner received any veterinary advice or treatment. A limitation of this study is that the measurement of how veterinary care was accessed relied on Dogslife owners' perception of what 'taking their dog to the vet' entails. SAVSNET reported an increase in phone consultations up to an average of about $3 \%$ of total consultations in the first and second phases of lockdown (23rd March - $10^{\text {th }}$ May 2020) (54). This initially seems like a small percentage and if this estimation is accurate for Dogslife dogs it does not explain the large reduction in veterinary visits reported by owners. However, SAVSNET acknowledge these figures are likely to be an underestimation due to differences in the workflow and data recording of veterinary practices (54). In support of this, the RCVS survey reported that $100 \%$ of 451 practices that answered the question used remote consulting for existing clients, whilst $45 \%$ used it for new clients (51). Furthermore, HealthforAnimals reported that in a survey of 3258 pet owners in the US, UK, France and Brazil, $27 \%$ delayed or avoided contacting their veterinarians and the percentage of owners whose veterinary practices offered digital or remote services rose from $20 \%$ to $47 \%$ (75). Similarly, a survey of veterinarians in California reported that practices providing telehealth services rose from $12 \%$ prior to the pandemic to $38 \%$ between March the $15^{\text {th }}$ and June the $15^{\text {th }} 2020(76)$. It is probable that the results in this study can be explained by a combination of Dogslife owners contacting their veterinarian less often due to concerns related to pandemic, experiencing an increase in telemedicine at their veterinary practices and having difficulties accessing veterinary care.

This study had several limitations which have not yet been discussed. We recommend that readers do not rely solely on the $p$-values reported to infer statistical significance and interpret our findings, but consider the confidence intervals and other results (49). Questionnaire data such as Dogslife is limited by social desirability bias (the tendency of survey and interview respondents to give answers they feel will be socially acceptable rather than those which reflect the truth) and 'recall decay' (the decrease in participants' ability to accurately recall events as time to reporting increases). Previous Dogslife studies have attempted to account for recall decay (77), but such methods introduce subjective cut-offs and limit the data available, which was not deemed appropriate in the current study. An arbitrary cut-off was used to allow for some delay in illness reporting by owners and it is unlikely to have fully captured the true number of illnesses reported that were experienced in the study period. However, this was used for both the COVID-19 restrictions study period and the data in the same date range in previous years, so it is unlikely that there was a bias in reporting that affected the comparison of these time periods.

Furthermore, attrition (the loss of participants during the course of the study) may have affected the results of this study. It is typical for some Dogslife owners to not report for a long period between one questionnaire and the next one. This makes it difficult to determine the true attrition rates for Dogslife and all owners were considered as 'available to report', when this was unlikely. The illnesses included in this study were reported by owners and had not received veterinary diagnoses, so they probably had a wide variety of aetiologies. Dogslife is a study of Labrador Retrievers and the results reported here may not be

Page 5/12 
generalisable to other dog breeds. Additionally, as has partly been discussed previously, the demography, dog owning experience and behaviour of Dogslife owners may differ from the general population of UK dog owners, especially during the COVID-19 restrictions study period with the increase of new dog owners. Finally, the cleaning, categorising and coding of the data included is subjective and relies on the researcher's expertise and opinion.

\section{Conclusion}

This study demonstrates that COVID-19 restrictions were associated with differences in Labrador Retrievers' lifestyle, routine care, insurance status, illness incidence and veterinary attendance who were living in England. Dogslife provided a unique opportunity to study prospective questionnaire data from owners already enrolled on a longitudinal cohort study, which is likely to minimise biases associated with recalling events prior to the pandemic. Furthermore, Dogslife includes a wider population of dogs than can be studied in primary care data and provides insights into owners' decision making about their dogs' healthcare. The implications of the changes to dogs' lives have not been fully realised, but future research should aim to elucidate how negative impacts of the COVID-19 pandemic and associated restrictions on dogs can be minimised.

\section{Methods}

\section{Collection and selection of Dogslife data}

Dogslife is a longitudinal online study of the health of pedigree UK Kennel Club registered Labrador Retrievers in the UK. Recruitment to Dogslife began in July 2010 and continues at the time of writing. Extensive details of the study design are published previously (59,78). Dogslife was approved by the University of Edinburgh Veterinary Ethical Review Committee (Ref: 7.5.09) and Human Ethical Review Committee (Ref: HERC_161_17). Dog owners register to Dogslife when their dogs are aged under one year and supply demographic and geographic information. They are then asked to complete online questionnaires about their dog's morphology, lifestyle and illness incidences every month when their dogs are under the age one and every three months when their dogs are over the age of one. All dogs were considered as 'available to report' at any time within the study.

Data for this study were collected from dogs living in England during the first 10 years of Dogslife, via routine online reporting. Dogs in other areas of the UK were not included into the study due to national differences in COVID-19 restrictions and because the majority of participants (80.02\%) in the Dogslife study live in England. Dogs in England were selected for inclusion into the study using owners' postcode data supplied at registration, which were classified into countries and checked for validity by joining to data publicly available from the Office for National Statistics (79). Data from the Office for National Statistics is licensed under the Open Government Licence V. 3.0.

The $23^{\text {rd }}$ of March 2020 was selected as the study onset date because it was when the first lockdown restrictions were introduced in England. The $4^{\text {th }}$ of July 2020 was selected as the study conclusion date because lockdown restrictions were eased considerably, resulting in the opening of public houses and restaurants and the beginning of localised lockdown restrictions in England (3). For clarity, this time period is referred to as the 'COVID-19 restrictions study period'. Data between the $23^{\text {rd }}$ of March and the $4^{\text {th }}$ of July in previous years from 2011 to 2019 were used as a comparison to data from the COVID-19 restrictions study period.

\section{Data cleaning and analysis}

Dogslife data was cleaned and assessed for quality prior to analysis and duplicates were removed while maximising the information they contained by filling in missing information. In categorical variables, where data was missing due to the Dogslife website auto-completion settings or where an owner had only partially completed a questionnaire, data was chronologically down-filled from the previous data entry, except when the first data entry and succeeding ones were missing, in which case data was chronologically up-filled from the nearest data entry point chronologically. Specific data cleaning of variables is discussed in more detail within the relevant methods sections. Additional research showed that the frequency with which owners' reported to Dogslife did not to differ in the COVID-19 restrictions study period in comparison with data in the same date range in previous years, so the potential of a confounding effect due to differences in reporting frequency was ruled out prior to other analyses [see Additional file 2].

All data analysis was carried out using R statistical software (R version 4.1.1). An example of the code, including the specific packages and functions used for this study are available in a public repository (80). All data are reported to four significant figures. The STrengthening the Reporting of OBservational studies in Epidemiology (STROBE) (81) reporting guidelines were adhered to in this study and a checklist was completed [see Additional file 3].

\section{Modelling the association of COVID-19 restrictions with the lifestyle and routine care of Dogslife dogs}

Dogslife questionnaire data was selected based on its perceived appropriateness to summarise the lifestyle and routine care of the cohort and transformed where necessary for modelling purposes into variables of interest (See Table 2). To transform exercise quantity from several categorical variables to a single continuous variable, a randomised value was assigned between the bottom and top limit of each time quantity category. For the category 'over 2 hours', a top limit of 4 hours was assumed. Weekday and weekend exercise quantities were combined by multiplying weekday quantities by $5 / 7$ and weekend quantities by $2 / 7$ and summing these values to produce a weighted average. The sum of all averaged exercise variables was calculated to produce a total 'exercise quantity' in minutes of per exercise week. Dried food was chosen as the indicator of food quantity because Dogslife owners report feeding dried food to their dog more frequently than other types of food and because the consistency and weight of dried food is less variable than wet food. Dried food quantity was cleaned using an adapted version of the published and validated 'NLME-A' method (82). In brief, non-linear mixed models were combined with an algorithm that used cut-off values based on recommendations for dried food intake for male and female Labrador Retriever puppies from the most popular dried food breed in Dogslife, which was Royal Canin (83). 
Various regression models were fitted to continuous variables of interest (exercise quantity and dried food quantity) and binomial variables of interest (insurance status, titbits status, sleep-person status, bathed SLV, anti-parasitic SLV, wormed SLV and vaccinated SLV). The final models chosen were GAMMs based on comparison of the models using various diagnostic techniques. Models for continuous variables of interest had Gaussian distributions and models for binomial variables of interest had binomial distributions with logit links. An independent variable was added to the models to compare the COVID-19 restrictions study period to data in the same date range in previous years. A smooth term for dog age was fitted to control for non-linear age effects, the sex of the dogs was included to control for sex effects and a random effects (RE) term was added for dog identification (ID) to control for individual effects. Dog age was calculated to the date that owners entered questionnaire data into the Dogslife website.

Table 2: Variables of interest derived from Dogslife questionnaires relating to lifestyle, routine care and insurance status

\begin{tabular}{|c|c|c|}
\hline $\begin{array}{l}\text { Name of } \\
\text { variable }\end{array}$ & Relevant Dogslife questionnaire question(s) and answer(s) reported by owners & Description of variable \\
\hline \multirow[t]{2}{*}{$\begin{array}{l}\text { Exercise } \\
\text { quantity }\end{array}$} & $\begin{array}{l}\text { Q: On average, in the last week for how long does [dog name] do the following exercise(s) EACH } \\
\text { day (Weekday/Weekend): Walking on the lead? Running on the lead? Walking/running off the } \\
\text { lead? Exercise involving fetching, chasing or retrieving? Obedience training? Other playing } \\
\text { activity (including dogs playing together)? }\end{array}$ & \multirow[t]{2}{*}{$\begin{array}{l}\text { Multiple categorical Dogslife } \\
\text { questions transformed to a single } \\
\text { continuous variable to estimate dogs } \\
\text { minutes of exercise per week }\end{array}$} \\
\hline & A: [None, $1-5$ mins, $5-15$ mins, $15-30$ mins, $30-60$ mins, $1-2$ hrs, Over 2 hrs] & \\
\hline \multirow{2}{*}{$\begin{array}{l}\text { Dried food } \\
\text { quantity }\end{array}$} & Q: How much dried food do you feed [dog name] in total each day? & \multirow{2}{*}{$\begin{array}{l}\text { Continuous variable converted into } \\
\text { grams of dried food fed to dogs per } \\
\text { day reported by owners }\end{array}$} \\
\hline & A: [Free text with unit box] & \\
\hline $\begin{array}{l}\text { Insurance } \\
\text { status }\end{array}$ & Q: Is [dog name] insured? A: [Yes/No] & Binomial yes/no variable \\
\hline $\begin{array}{l}\text { Titbits } \\
\text { status }\end{array}$ & $\begin{array}{l}\text { Q: Does [dog name] also receive 'titbits'? For example anything else [dog name] eats such as } \\
\text { food off your plate, training treats, chews etc. A: [Yes/No] }\end{array}$ & Binomial yes/no variable \\
\hline \multirow{2}{*}{$\begin{array}{l}\text { Sleep- } \\
\text { person } \\
\text { status }\end{array}$} & Q: Where does [dog name] sleep at night? & \multirow{2}{*}{$\begin{array}{l}\text { Transformed into a single binomial } \\
\text { yes/no variable to categorise whether } \\
\text { dogs sleep with a person at night }\end{array}$} \\
\hline & $\begin{array}{l}\text { A: [Alone in a room in a house, In a room shared with a person, In a room shared with a pet, In a } \\
\text { room shared with a pet and a person, Outside, Other] }\end{array}$ & \\
\hline $\begin{array}{l}\text { Bathed } \\
\text { SLV* }^{*}\end{array}$ & Q: Has [dog name] been bathed since you last visited the site? A: [Yes/No] & Binomial yes/no variable \\
\hline $\begin{array}{l}\text { Anti- } \\
\text { parasitic } \\
\text { SLV* }\end{array}$ & $\begin{array}{l}\text { Q: Have you used any products to prevent or treat fleas or ticks since you last visited the site? A: } \\
\text { [Yes/No] }\end{array}$ & Binomial yes/no variable \\
\hline $\begin{array}{l}\text { Wormed } \\
\text { SLV* }^{*}\end{array}$ & Q: Has [dog name] been wormed since you last visited the site? A: [Yes/No] & Binomial yes/no variable \\
\hline $\begin{array}{l}\text { Vaccinated } \\
\text { SLV* }\end{array}$ & Q: Has [dog name] been vaccinated since you last visited the site? A: [Yes/No] & Binomial yes/no variable \\
\hline
\end{tabular}

*Since the owner's last visit to the Dogslife website (SLV).

\section{Analysing the association of COVID-19 restrictions with illness incidence and associated veterinary attendance in Dogslife}

Dogslife questionnaire data was selected that related to dogs' illness incidences, the start dates of illness and dogs' veterinary attendance with illness incidences (See Table 3). When illness start dates were suspected to be erroneous (E.g. when the date was before the dog's date of birth), they were corrected (E.g. when the date was suspected to be a unit error away from the correct date) or removed.

The incidences of illness and veterinary attendance with an illness were first investigated as a total count of questionnaires where owners reported any illness or any veterinary attendance with an illness respectively and illnesses were then further classified into ten categories. The first six illness categories were routinely reported by owners in each Dogslife questionnaire: vomiting, diarrhoea, coughing, scratching, licking or chewing and limping or lameness. The final four illness categories were derived from the 'other illness' category of Dogslife questionnaires. The primary researcher (CSCW) manually coded other illnesses and the four most frequently reported were selected for inclusion into the study: eye problems, ear problems, skin problems and accident or injury.

From the beginning of Dogslife in July 2010 to the $1^{\text {st }}$ of August 2020, there was a median reporting time lag of 19 days (Interquartile range: 35 ) between the illness start dates given by owners and the date that owners entered questionnaire data into the Dogslife database. To allow for some owner delay in illness reporting, 19 days were added onto the study conclusion date (the $4^{\text {th }}$ of July became the $23^{\text {rd }}$ of July in each year) and questionnaires were included up to this date. Where the illness start dates given by owners were available (85.69\%), they were used to exclude illnesses that were before the $23^{\text {rd }}$ of March and after the $4^{\text {th }}$ of July from 2011 to 2020 .

Table 3: Information derived from Dogslife questionnaires relating to dogs' illness and veterinary attendance status 


Relevant Dogslife questionnaire question(s)
Q: Has [dog name] had any of the following problems: Vomiting? Diarrhoea? Coughing? Scratching themselves? Licking or IIIness incidences
chewing themselves? Limping or lameness? Did [dog name] have any other illnesses or problems?
A: [Yes/No]
Q. Approximately when did the vomiting start?
A: [Calendar box]
Q. Did you take [dog name] to your vet for the [illness type]?
A: [Yes/No]

Chi-squared tests were performed to compare the incidences of illness and associated veterinary attendance in the COVID-19 restrictions study period to data in the same date range in previous years. To account for the aging population of the Dogslife cohort, these tests were performed with age stratification using the $\mathrm{MH}$ adjustment into the following four age groups: under 1 year, 1 to 3.49 years, 3.5 to 6.99 years and 7 years or over. The age groups were arbitrarily defined to separate dogs under one year due to differences in Dogslife reporting, to divide the remaining data within the confines of Dogslife (where the oldest dog was less than 11 years old) and to approximately capture the four periods of Labrador Retriever aging as reported by Wang and colleagues (84). The MH adjustment was not performed when the expected count for a stratified group was $<5$, as chi-square tests are inaccurate at small numbers of expected frequencies $(85,86)$. Dog age was calculated to the illness start dates given by owners where they were available (85.69\%) and otherwise to the date that owners entered questionnaire data into the Dogslife database.

\section{Declarations}

\section{Ethics approval and consent to participate}

The study protocol was in accordance with the ethics guidelines of the University of Edinburgh and used data from Dogslife, which was approved by the University of Edinburgh Veterinary Ethical Review Committee (Ref: 7.5.09) and Human Ethical Review Committee (Ref: HERC_161_17). All dog owners in the Dogslife study gave their informed consent to participate upon signing up to the online questionnaire.

\section{Consent for publication}

Not applicable.

\section{Availability of data and materials}

The datasets generated and/or analysed during the current study are not publicly available because individual privacy may be compromised but are available from the corresponding author on reasonable request.

\section{Competing interests}

The authors declare that they have no competing interests.

\section{Funding}

This work was supported by an Institute Strategic Programme Grant from the Biotechnology and Biological Sciences Research Council (https://bbsrc.ukri.org/) to the Roslin Institute [BB/ J004235/1] and the lead author was funded by the Biotechnology and Biological Sciences Research Council under the EASTBIO (http://www.eastscotbiodtp.ac.uk/) doctoral training programme [BB/ J01446X/1 to CSCW]. The funding sources had no role in the data collection, design, analysis, interpretation of data, reporting or writing of the study.

\section{Authors' contributions}

CSCW is guarantor and was responsible for planning and conceptualisation, funding acquisition, data curation and cleaning, statistical analysis, data visualisation, methodological design and validation, experimental investigation, writing programmatic code and drafting and revising the paper. IGH was involved in planning and conceptualisation, funding acquisition, supervision, methodological design and revising the paper. BMB was involved in planning and conceptualisation, funding acquisition, supervision, methodological design and revising the paper. JJS was involved in planning and conceptualisation, funding acquisition, supervision, methodological design and revising the paper. DNC was involved in project administration, obtaining resources, planning and conceptualisation, funding acquisition, supervision, methodological design and revising the paper.

\section{Acknowledgements}

The authors are grateful for the participants who contributed and continue to contribute data to Dogslife.

\section{References}

1. Zhu H, Wei L, Niu P. The novel coronavirus outbreak in Wuhan, China. Glob Heal Res Policy. 2020;5(6). 
2. Hale T, Angrist N, Goldszmidt R, Kira B, Petherick A, Phillips T, et al. A global panel database of pandemic policies (Oxford COVID-19 Government Response Tracker). Nat Hum Behav. 2021;5(4):529-38.

3. Dunn P, Allen L, Cameron G, Alderwick H. COVID-19 policy tracker. [Internet]. The Health Foundation. 2020 [cited 2021 Oct 1]. Available from: https://www.health.org.uk/\%0Anews-and-comment/charts-and-infographics/covid-19-policy-tracker.

4. Pet Food Manufacturers' Association. Pet Population 2021 [Internet]. Pet Food Manufacturers' Association. 2021 [cited 2021 Dec 9]. Available from: https://www.pfma.org.uk/pet-population-2021.

5. Morgan L, Protopopova A, Birkler RID, Itin-Shwartz B, Sutton GA, Gamliel A, et al. Human-dog relationships during the COVID-19 pandemic: booming dog adoption during social isolation. Humanit Soc Sci Commun. 2020;7(1):1-11.

6. Ho J, Hussain S, Sparagano O. Did the COVID-19 Pandemic Spark a Public Interest in Pet Adoption? Front Vet Sci. 2021;8(647308).

7. Packer RMA, Brand CL, Belshaw Z, Pegram CL, Stevens KB, O’Neill DG. Pandemic puppies: Characterising motivations and behaviours of UK owners who purchased puppies during the 2020 COVID-19 pandemic. Animals. 2021;11(9).

8. Hargrave C. COVID-19: implications of self-isolation and social distancing for the emotional and behavioural health of equines, parrots and small prey pets. Companion Anim. 2020;25(4):1-6.

9. Jalongo MR. Pet Keeping in the Time of COVID-19: The Canine and Feline Companions of Young Children. Early Child Educ J. 2021;18.

10. World Health Organization. COVID-19 clinical management: living guidance, 25 January 2021 [Internet]. World Health Organization. World Health Organization; 2021 [cited 2021 Dec 1]. Available from: https://apps.who.int/iris/handle/10665/338882.

11. Perico N, Suter F, Remuzzi G. A recurrent question from a primary care physician: How should I treat my COVID-19 patients at home? Clin Med Investig. 2020;5(2).

12. Adamelli S, Tocchio A, Brini C. COVID-19 Pandemic and Rescue of Pets. The Role of Veterinarians in the Human-Animal-Environment Relationship at the Time of the Coronavirus. J Appl Anim Ethics Res. 2021;3(1):91-102.

13. Applebaum JW, Adams BL, Eliasson MN, Zsembik BA, McDonald SE. How pets factor into healthcare decisions for COVID-19: A One Health perspective. One Heal. 2020;11.

14. Kogan LR, Erdman P, Bussolari C, Currin-McCulloch J, Packman W. The Initial Months of COVID-19: Dog Owners' Veterinary-Related Concerns. Front Vet Sci. 2021;8.

15. Owczarczak-Garstecka SC, Graham TM, Archer DC, Westgarth C. Dog walking before and during the COVID-19 pandemic lockdown: Experiences of UK dog owners. Int J Environ Res Public Health. 2021;18(12).

16. Pieh C, Budimir S, Delgadillo J, Barkham M, Fontaine JRJ, Probst T. Mental Health during COVID-19 Lockdown in the United Kingdom. Psychosom Med. 2021;83(4):328-37.

17. Nochaiwong S, Ruengorn C, Thavorn K, Hutton B, Awiphan R, Phosuya C, et al. Global prevalence of mental health issues among the general population during the coronavirus disease-2019 pandemic: a systematic review and meta-analysis. Sci Rep. 2021;11(1).

18. Kaufman KR, Petkova E, Bhui KS, Schulze TG. A global needs assessment in times of a global crisis: world psychiatry response to the COVID-19 pandemic. BJPsych open. 2020;6(3).

19. Bussolari C, Currin-Mcculloch J, Packman W, Kogan L, Erdman P. “I Couldn't Have Asked for a Better Quarantine Partner!": Experiences with Companion Dogs during Covid-19. Animals. 2021;11(330).

20. Hawkins RD, Brodie ZP. The role of human- pet attachment on people's mental health over time during COVID-19 lockdown. In: NRS Mental Health 2020 Annual Scientific Meeting, United Kingdom. 2020.

21. Ratschen E, Shoesmith E, Shahab L, Silva K, Kale D, Toner P, et al. Human-animal relationships and interactions during the Covid-19 lockdown phase in the UK: Investigating links with mental health and loneliness. PLoS One. 2020;15(9):e0239397.

22. Applebaum JW, Tomlinson CA, Matijczak A, McDonald SE, Zsembik BA. The concerns, difficulties, and stressors of caring for pets during covid-19: Results from a large survey of U.S. pet owners. Animals. 2020;10(1882).

23. Ryan MG, Storey AE, Anderson RE, Walsh CJ. Physiological Indicators of Attachment in Domestic Dogs (Canis familiaris) and Their Owners in the Strange Situation Test. Front Behav Neurosci. 2019;13(162).

24. Sümegi Z, Oláh K, Topál J. Emotional contagion in dogs as measured by change in cognitive task performance. Appl Anim Behav Sci. 2014;160:10615.

25. Sundman AS, Van Poucke E, Svensson Holm AC, Faresjö $\AA$, Theodorsson E, Jensen P, et al. Long-term stress levels are synchronized in dogs and their owners. Sci Rep. 2019;9(7391).

26. Yong MH, Ruffman T. Emotional contagion: Dogs and humans show a similar physiological response to human infant crying. Behav Processes. 2014;108:155-65.

27. Piotti P, Karagiannis $C$, Satchell L, Michelazzi M, Albertini M, Alleva E, et al. Use of the Milan pet quality of life instrument (MPQL) to measure pets' quality of life during COVID-19. Animals. 2021;11(1336).

28. Shoesmith E, de Assis LS, Shahab L, Ratschen E, Toner P, Kale D, et al. The perceived impact of the first UK COVID-19 lockdown on companion animal welfare and behaviour: A mixed-method study of associations with owner mental health. Int J Environ Res Public Health. 2021;18(6171).

29. Janssen X, Fleming L, Kirk A, Rollins L, Young D, Grealy M, et al. Changes in physical activity, sitting and sleep across the COVID-19 national lockdown period in Scotland. Int J Environ Res Public Health. 2020;17(9362).

Page 9/12 
30. Okely JA, Corley J, Welstead M, Taylor AM, Page D, Skarabela B, et al. Change in physical activity, sleep quality, and psychosocial variables during COVID-19 lockdown: Evidence from the lothian birth cohort 1936. Int J Environ Res Public Health. 2021;18(210).

31. Robertson M, Duffy F, Newman E, Prieto Bravo C, Ates HH, Sharpe H. Exploring changes in body image, eating and exercise during the COVID-19 lockdown: A UK survey. Appetite. 2021;159(105062).

32. Naughton F, Ward E, Khondoker M, Belderson P, Marie Minihane A, Dainty J, et al. Health behaviour change during the UK COVID-19 lockdown: Findings from the first wave of the C-19 health behaviour and well-being daily tracker study. Br J Health Psychol. 2021;26(2):624-43.

33. Robinson E, Boyland E, Chisholm A, Harrold J, Maloney NG, Marty L, et al. Obesity, eating behavior and physical activity during COVID-19 lockdown: A study of UK adults. Appetite. 2021;156(104853).

34. Office for National Statistics. Coronavirus (COVID-19) latest insights: Work [Internet]. Office for National Statistics. 2021 [cited 2021 Nov 12]. Available from:

https://www.ons.gov.uk/peoplepopulationandcommunity/healthandsocialcare/conditionsanddiseases/articles/coronaviruscovid19latestinsights/work.

35. Heirs M, Graham H. Canine separation anxiety: how first referral practices can help. Companion Anim. 2021;26(8):170-4.

36. Vučinić M, Vučićević M, Nenadović K. The Covid-19 Pandemic Affects Owners Walking With Their Dogs. J Vet Behav. 2021;48.

37. Angelo D, Chirico A, Sacchettino L, Manunta F, Martucci M, Cestaro A, et al. Human-Dog Relationship during the First COVID-19 Lockdown in Italy. Animals. 2021;11(2335).

38. Esam F, Forrest R, Waran N. Locking down the impact of New Zealand's COVID-19 alert level changes on pets. Animals. 2021;11(758).

39. Martos Martinez-Caj A, De Herdt V, Enders Slegers MJ, Moons CPH. Pets and their owners during the first COVID-19 lockdown period: perceived changes in routines and emotions - an exploratory study. J Vet Behav. 2021;In Press.

40. Christley RM, Murray JK, Anderson KL, Buckland EL, Casey RA, Harvey ND, et al. Impact of the first COVID-19 lockdown on management of pet dogs in the UK. Animals. 2021;11(5).

41. Payne E, DeAraugo J, Bennett P, McGreevy P. Exploring the existence and potential underpinnings of dog-human and horse-human attachment bonds. Behav Processes. 2016;125:114-21.

42. Vincent A, Mamzer H, Ng Z, Farkas KJ. People and Their Pets in the Times of the Covid-19 Pandemic. Soc Regist. 2020;4(3):111-28.

43. British Veterinary Association. BVA response to Animal Welfare Committee: Call for information on animal welfare issues related to Covid-19 restrictions [Internet]. British Veterinary Association. 2020 [cited 2021 Nov 5]. Available from: https://www.bva.co.uk/media/3651/bva-response-to-awc-coronavirus-inquiry-final.pdf.

44. Holland KE, Owczarczak-Garstecka SC, Anderson KL, Casey RA, Christley RM, Harris L, et al. "More Attention than Usual”: A Thematic Analysis of Dog Ownership Experiences in the UK during the First COVID-19 Lockdown. Animals. 2021;11(240).

45. Dogs Trust. The impact of COVID-19 lockdown restrictions on dogs \& dog owners in the UK [Internet]. Dogs Trust. 2020 [cited 2021 Sep 17]. Available from: https://www.dogstrust.org.uk/help-advice/research/research-papers/201020_covid report_v8.pdf.

46. Jezierski T, Camerlink I, Peden RSE, Chou JY, Marchewka J. Changes in the health and behaviour of pet dogs during the COVID-19 pandemic as reported by the owners. Appl Anim Behav Sci. 2021;241(105395).

47. Bowen J, García E, Darder P, Argüelles J, Fatjó J. The effects of the Spanish COVID-19 lockdown on people, their pets, and the human-animal bond. J Vet Behav. 2020;40:75-91.

48. Parente G, Gargano T, Di Mitri M, Cravano S, Thomas E, Vastano M, et al. Consequences of COVID-19 lockdown on children and their pets: Dangerous increase of dog bites among the paediatric population. Children. 2021;8(620).

49. British Veterinary Association. Guidance for veterinary practices in providing essential veterinary care during the Covid-19 pandemic [Internet]. British Veterinary Association. 2020 [cited 2021 Nov 5]. Available from: https://www.bva.co.uk/media/3434/bva-guidance-for-veterinary-practices-on-covid-19from-14-april-2020-final.pdf.

50. Van Dobbenburgh R, De Briyne N. Impact of Covid-19 on animal welfare. Vet Rec. 2020;187(5).

51. Royal College of Veterinary Surgeons. Results from RCVS survey \#1 (carried out between 3-7 April 2020) on the economic impact of Covid-19 on veterinary clinical practices [Internet]. Royal College of Veterinary Surgeons. 2020 [cited 2021 Nov 4]. Available from: https://www.rcvs.org.uk/news-andviews/publications/coronavirus-economic-impact-on-veterinary-practice/.

52. The Small Animal Veterinary Surveillance Network. Impact of Covid-19 on Companion Animal Veterinary Practice. Report 1, 20 April 2020 [Internet]. The Small Animal Veterinary Surveillance Network at the University of Liverpool. 2020 [cited 2021 Oct 31]. Available from:

https://www.liverpool.ac.uk/media/livacuk/savsnet/Impact,of,COVID-19,on,companion,animal,veterinary,practice,report,1,20apr20.pdf.

53. The Small Animal Veterinary Surveillance Network. Impact of Covid-19 on Companion Animal Veterinary Practice. Report 2, 5th May 2020 [Internet]. The Small Animal Veterinary Surveillance Network at the University of Liverpool. 2020 [cited 2021 Oct 31]. Available from:

https://www.liverpool.ac.uk/media/livacuk/savsnet/Impact,of,COVID-19,on,companion,animal,veterinary,practice,report,2.pdf.

54. The Small Animal Veterinary Surveillance Network. Impact of Covid-19 on Companion Animal Veterinary Practice. Report 6, 16th December 2020 [Internet]. The Small Animal Veterinary Surveillance Network at the University of Liverpool. 2020 [cited 2021 Oct 31]. Available from: https://www.liverpool.ac.uk/media/livacuk/savsnet/Impact,of,COVID-19,on,companion,animal,veterinary,practice,report,6.pdf.

55. Elnegaard S, Andersen R, Pedersen A, Larsen P, Søndergaard J, Rasmussen S, et al. Self-reported symptoms and healthcareseeking in the general population - exploring "The Symptom Iceberg”. BMC Public Health. 2015;15(685). 
56. Smith SL, Singleton DA, Noble PJ, Radford AD, Brant B, Pinchbeck GL, et al. Possible cause of outbreak of prolific vomiting in dogs. Vet Rec. 2020;186(10):324.

57. Singleton DA, Noble PJ, Radford AD, Brant B, Pinchbeck GL, Greenberg D, et al. Prolific vomiting in dogs. Vet Rec. 2020;186(6):191.

58. Woolley CSC, Handel IG, Bronsvoort BM, Schoenebeck JJ, Clements DN. Surveillance of a vomiting outbreak in dogs in the UK using owner-derived and internet search data. Vet Rec. 2021 Nov;189(9):e308.

59. Clements DN, Handel IG, Rose E, Querry D, Pugh CA, Ollier WER, et al. Dogslife: a web-based longitudinal study of Labrador Retriever health in the UK. BMC Vet Res. 2013;9(13).

60. Kelly M. Over a third of dog owners admit their pampered pets are overweight [Internet]. ChronicleLive. 2021 [cited 2021 Nov 10]. Available from: https://www.chroniclelive.co.uk/news/north-east-news/dog-owners-pets-overweight-lockdown-20323481.

61. Butterworth B. Dogs have put on half a stone in lockdown after too many treats, research finds [Internet]. iNews. 2021 [cited 2021 Nov 15 ]. Available from: https://inews.co.uk/news/uk/dogs-put-on-weight-lockdown-too-many-treats-research-finds-944041.

62. Delgado K. Dogs are getting overweight in lockdown - here's how to give them a new leash of life [Internet]. iNews. [cited 2021 Nov 11]. Available from: https://inews.co.uk/news/long-reads/dogs-overweight-lockdown-how-to-lose-weight-portion-control-outdoors-865133.

63. Morelli G, Marchesini G, Contiero B, Fusi E, Diez M, Ricci R. A Survey of Dog Owners' Attitudes toward Treats. J Appl Anim Welf Sci. 2020 Jan 2;23(1).

64. White GA, Ward L, Pink C, Craigon J, Millar KM. "Who's been a good dog?” - Owner perceptions and motivations for treat giving. Prev Vet Med. 2016;132:14-9.

65. Kuhl CA, Dean R, Quarmby C, Lea RG. Information sourcing by dog owners in the UK: Resource selection and perceptions of knowledge. Vet Rec. $2021 ; \mathrm{e} 1081$.

66. Elsheikha HM. Pet worming protocols: how to ensure owner compliance. VNTimes. 2016;16(7):8-12.

67. Stokes L, Wright I. Parasites in the UK: trends and themes of 2020. Companion Anim. 2021;26(3):62-5.

68. Loeb J. 'Most vaccinations should not be carried out'. Vet Rec. 2020;186(13):398.

69. The Small Animal Veterinary Surveillance Network. Impact of Covid-19 on Companion Animal Veterinary Practice. Report 4, 15th July 2020 [Internet]. The Small Animal Veterinary Surveillance Network at the University of Liverpool. 2020 [cited 2021 Oct 31]. Available from:

https://www.liverpool.ac.uk/media/livacuk/savsnet/Impact,of,COVID-19,on,companion,animal,veterinary,practice,report,4.pdf.

70. World Health Organization. Global progress against measles threatened amidst COVID-19 pandemic [Internet]. World Health Organization. 2021 [cited 2021 Nov 17]. Available from: https://www.who.int/news/item/10-11-2021-global-progress-against-measles-threatened-amidst-covid-19-pandemic.

71. Day MJ. Small animal vaccination: A practical guide for vets in the UK. In Pract. 2017;39(3):110-8.

72. GoCompare. Coronavirus hits pet industry as dog prices boom during lockdown [Internet]. GoCompare. 2020 [cited 2021 Nov 17]. Available from: https://www.gocompare.com/pet-insurance/coronavirus-hits-pet-industry/.

73. Buonavoglia C, Martella V. Canine respiratory viruses. Vet Res. 2007;38(2):355-73.

74. Maboni G, Seguel M, Lorton A, Berghaus R, Sanchez S. Canine infectious respiratory disease: New insights into the etiology and epidemiology of associated pathogens. 2019;14(4):e0215817.

75. HealthforAnimals. Survey of Pet Owners Shows Impacts of COVID-19 Pandemic on Veterinary Care [Internet]. HealthforAnimals. 2021 [cited 2021 Nov 12]. p. 19-22. Available from: https://www.healthforanimals.org/resources/newsletter/articles/survey-of-pet-owners-shows-impacts-of-covid-19pandemic-on-veterinary-care/.

76. Dubin RJ, Angliss G, Eng C, Cisneros T, Griffon D. Veterinarians' perceptions of COVID-19 pandemic-related influences on veterinary telehealth and on pet owners' attitudes toward cats and dogs. Small Anim Exot. 2021;259(10).

77. Pugh CA, Summers KM, Bronsvoort BM, Handel IG, Clements DN. Validity of internet-based longitudinal study data: The elephant in the virtual room. J Med Internet Res. 2015;17(4).

78. Pugh CA, Bronsvoort BM, Handel IG, Summers KM, Clements DN. Dogslife: A cohort study of Labrador Retrievers in the UK. Prev Vet Med. 2015;122(4):426-35.

79. Office for National Statistics. National Statistics Postcode Lookup UK (February 2020); Contains Ordnance Survey data @ Crown copyright and database right 2020. [Internet]. Office for National Statistics. 2020 [cited 2020 Mar 6]. Available from:

https://geoportal.statistics.gov.uk/datasets/1951e70c3cc3483c9e643902d858355b/.

80. Woolley C. COVID19 restrictions impact on Dogslife [Internet]. Github. 2021 [cited 2021 Dec 28]. Available from: https://github.com/CharlotteWoolley/COVID19_restrictions_impact_on_Dogslife.

81. von Elm E, Altman DG, Egger M, Pocock SJ, Gotzsche PC, Vandenbroucke JP. The Strengthening the Reporting of Observational Studies in Epidemiology (STROBE) Statement: guidelines for reporting observational studies. PLoS Med. 2007;4(10):e296.

82. Woolley CSC, Handel IG, Bronsvoort BM, Schoenebeck JJ, Clements DN. Is it time to stop sweeping data cleaning under the carpet? A novel algorithm for outlier management in growth data. PLoS One. 2020;15(1):e0228154.

83. Royal Canin. Puppy Chiot Labrador Retriever Feeding Guide. [Internet]. Royal Canin. [Undated]. [cited 2020 Mar 1]. Available from: https://www.royalcanin.com/ca/en_ca/-/media/royal-canin/canada/images/products/dogs/labrador-retriever-puppy-dry-dogfood/bwn6fr2lwq8hfcdmlfgh.ashx.

84. Wang T, Ma J, Hogan AN, Fong S, Licon K, Tsui B, et al. Quantitative Translation of Dog-to-Human Aging by Conserved Remodeling of the DNA Methylome. Cell Syst. 2020;11(2):176-185.e6. 
85. Yates D, Moor D, McCabe G. The Practice of Statistics. 1st Ed. New York: Freeman; 1999.

86. Cochran WG. The [chi-squared] test of goodness of fit. Ann Math Stat. 1952;25:315-345.

\section{Figures}

\section{Figure 1}

Forest plot of estimates from generalised additive mixed models

Contains the number of Dogslife questionnaires with data $(\mathrm{N})$, the models' type (linear or logistic), the models' estimates for the beta coefficients (for linear models) or odds ratios (for logistic models) and associated 95\% confidence intervals (CI) and P-values for comparing the COVID-19 restrictions study period (March $23^{\text {rd }}$ to July $4^{\text {th }} 2020$ ) to data in the same date range in previous years (March $23^{\text {rd }}$ to July $4^{\text {th }}, 2010$ to 2019 ) in each of the variables of interest. All models were adjusted for age, sex and individual effects. *Since the owner's last visit to the Dogslife website (SLV).

\section{Figure 2}

Forest plot of estimates from chi-squared tests

Contains the age-adjusted chi-squared tests' estimates for the odds ratios and associated $95 \%$ confidence intervals (Cl) and P-values for comparing the Dogslife illness incidences and veterinary attendance (VA) with illness incidences in the COVID-19 restrictions study period (March 23 rd to July $4^{\text {th }}$ 2020) to data in the same date range in previous years (March 23rd to July $4^{\text {th }}, 2010$ to 2019). *The Mantel-Haenszel adjustment for age was not performed on these variables due to expected frequencies of the stratified groups containing values less than 5 .

\section{Supplementary Files}

This is a list of supplementary files associated with this preprint. Click to download.

- Additionalfile1.pdf

- Additionalfile2.pdf

- Additionalfile3.pdf 